\title{
Using Machine Learning-Based Approaches for the Detection and Classification of Human Papillomavirus Vaccine Misinformation: Infodemiology Study of Reddit Discussions
}

Jingcheng $\mathrm{Du}^{1 *}, \mathrm{PhD}$; Sharice Preston ${ }^{2 *}, \mathrm{PhD}$; Hanxiao Sun ${ }^{1,2}, \mathrm{MSc}$; Ross Shegog ${ }^{2}, \mathrm{PhD}$; Rachel Cunningham ${ }^{3}, \mathrm{MSc}$; Julie Boom ${ }^{3,4}$, MD; Lara Savas ${ }^{2}, \mathrm{PhD}$; Muhammad Amith ${ }^{1}, \mathrm{PhD}$; Cui Tao ${ }^{1}, \mathrm{PhD}$

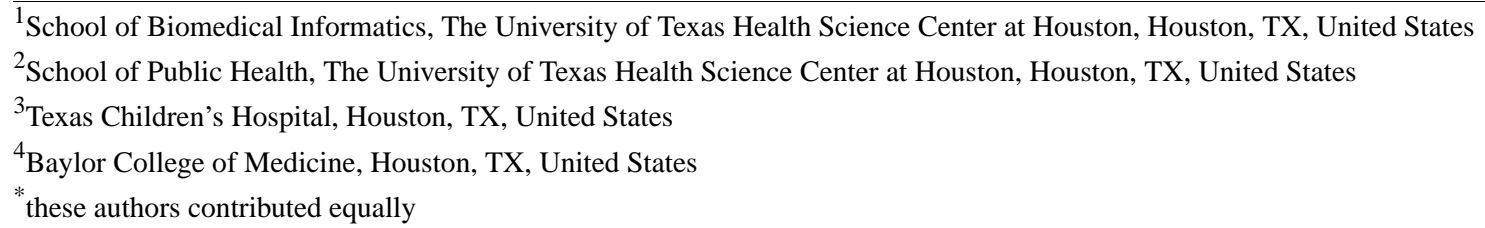

\section{Corresponding Author:}

Cui Tao, $\mathrm{PhD}$

School of Biomedical Informatics

The University of Texas Health Science Center at Houston

7000 Fannin St

\#600

Houston, TX, 77030

United States

Phone: 1713500981

Email: cui.tao@uth.tmc.edu

\section{Abstract}

Background: The rapid growth of social media as an information channel has made it possible to quickly spread inaccurate or false vaccine information, thus creating obstacles for vaccine promotion.

Objective: The aim of this study is to develop and evaluate an intelligent automated protocol for identifying and classifying human papillomavirus (HPV) vaccine misinformation on social media using machine learning (ML)-based methods.

Methods: Reddit posts (from 2007 to 2017, N=28,121) that contained keywords related to HPV vaccination were compiled. A random subset $(2200 / 28,121,7.82 \%)$ was manually labeled for misinformation and served as the gold standard corpus for evaluation. A total of $5 \mathrm{ML}$-based algorithms, including a support vector machine, logistic regression, extremely randomized trees, a convolutional neural network, and a recurrent neural network designed to identify vaccine misinformation, were evaluated for identification performance. Topic modeling was applied to identify the major categories associated with HPV vaccine misinformation.

Results: A convolutional neural network model achieved the highest area under the receiver operating characteristic curve of 0.7943. Of the 28,121 Reddit posts, 7207 (25.63\%) were classified as vaccine misinformation, with discussions about general safety issues identified as the leading type of misinformed posts (2666/7207, 36.99\%).

Conclusions: ML-based approaches are effective in the identification and classification of HPV vaccine misinformation on Reddit and may be generalizable to other social media platforms. ML-based methods may provide the capacity and utility to meet the challenge involved in intelligent automated monitoring and classification of public health misinformation on social media platforms. The timely identification of vaccine misinformation on the internet is the first step in misinformation correction and vaccine promotion.

(J Med Internet Res 2021;23(8):e26478) doi: 10.2196/26478

\section{KEYWORDS}

HPV vaccine; social media; misinformation; infodemiology; infoveillance; deep learning; Reddit; machine learning 


\section{Introduction}

\section{Background}

Human papillomavirus (HPV) infection is a highly prevalent sexually transmitted infection. HPV infections cause approximately 33,700 cases of cancer every year in the United States, including cervical, vaginal, penile, anal, and head and neck cancers [1,2]. Since 2006, a vaccine against the most common HPV subtypes has been available to prevent associated cancers and genital warts [3]. Despite undeniable evidence of its effectiveness, the HPV vaccine has been controversial among parents, which has contributed to vaccine hesitancy and even refusal [4] and to relatively low national rates of HPV vaccine initiation and series completion [5]. Resistance to the HPV vaccine has been a result of parents' concerns about the vaccine's effect on sexual behavior, because HPV is a sexually transmitted infection, and the safety of the vaccine, as well as inconsistent vaccine recommendations from health care providers [6].

A burgeoning antivaccine movement has affected overall vaccine coverage in the United States and contributed to a resurgence of vaccine-preventable diseases such as measles [7]. Vaccine hesitancy has been found to be driven mainly by concerns about vaccine safety and is propelled by misinformation circulated through social media [8]. The rapid growth of social media as an information channel has made it possible to quickly spread inaccurate or false information and create a platform for antivaccine campaigns to promulgate vaccine-related misinformation [9]. Participants in the antivaccine movement circulate antivaccine sentiments and misinformation through various internet channels and create demonstrable impact on individual and community health [10]. Experts in media communications have suggested that web-based misinformation is becoming unmanageable, even as concern increases about the damage it causes to consumer well-being [11]. Efforts to curtail the phenomenon, such as story-flagging and fact-checking tools, are not enough to suppress the advocates of misinformation $[12,13]$ because the efficiency and scalability of these tools are limited, and misinformation is disseminated much faster and broader than true information.

Mitigation of medical and public health misinformation on social media is important; however, the sheer amount of information makes it challenging to identify these posts efficiently and accurately. Although social media is a convenient way for users to generate, share, receive, and comment on social content [14], there is a need for broad-scale, innovative methods to track and understand the spread of health misinformation on social media outlets [15].
Identifying vaccine-related misinformation presented on social media is an important first step in the timely curbing of the ongoing spread of vaccine misinformation. Given the large volume of social media posts and unique features of social media language (ie, incomplete sentences and misspellings), the use of automated methods for the identification of misinformation is challenging. However, machine learning (ML)-based approaches have been previously applied to identify misinformation on Twitter regarding controversial topic domains [16] and rumors regarding a range of topics [17]. ML involves the use of algorithms and statistical modeling that provide the ability to automatically conduct tasks and learn without using explicit programming [18]. Despite the utility of these ML approaches, there is a dearth of application to medical or health topics. To date, an ML-based system has tracked misinformation about the Zika virus on social media [19] and classified misinformation within specific health forums (eg, MedHelp) [20]. Although there have been efforts to develop ML for sentiment analysis on vaccine topics [21,22], to our knowledge, there is no prior work on automated identification of vaccine-related misinformation on social media. Deep learning (DL) is a subset of ML algorithms based on deep neural networks. Although DL has advanced ML algorithms in multiple tasks [23], the utility of DL regarding vaccine misinformation identification is still unclear.

\section{Objective}

We report the utility of various conventional ML and DL algorithms to automatically identify and categorize misinformation on the HPV vaccine using posts on Reddit, a popular social media platform with more than 330 million monthly active users [24]. Reddit users are primarily anonymous young users below the age of 35 years, and more than half (54\%) live in the United States [25]). Studies have revealed that young adults in the United States have low perceived susceptibility of contracting HPV [26], low health literacy pertaining to HPV and the HPV vaccine [27], and are more likely to seek health information on social media than other age demographics [28]. Table S1 in Multimedia Appendix 1 lists ML-related terms in the manuscript and their definitions.

\section{Methods}

\section{Overview}

We used a hybrid approach for the identification and classification of HPV vaccine misinformation on Reddit (Figure 1). Our approach can be divided into two steps: (1) evaluation of ML algorithms for vaccine misinformation identification and (2) topic modeling on Reddit posts that contain vaccine misinformation (ML-inferred). 
Figure 1. The overview of human papillomavirus misinformation identification and classification on Reddit. (a) Evaluation of machine learning-based misinformation identification and (b) topic modeling. ML: machine learning.

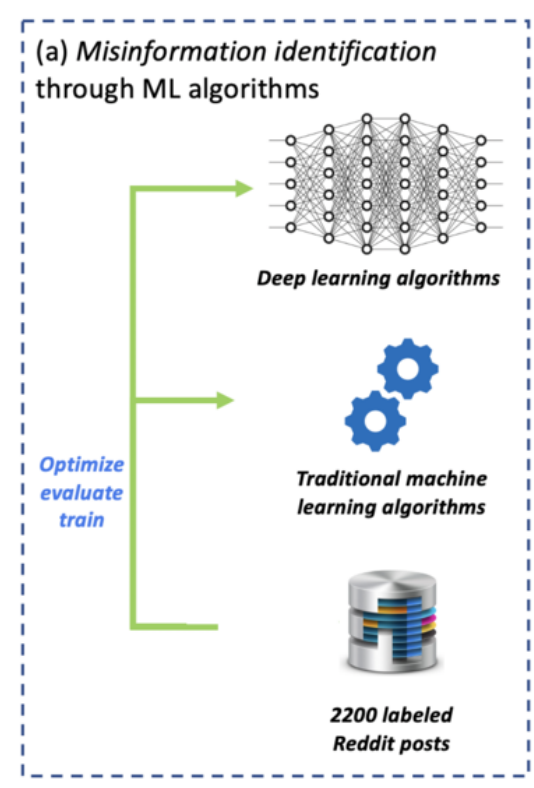

Reddit posts that contain HPV vaccine keywords were first collected, after which random subsets of the posts were labeled as containing misinformation or nonmisinformation. The labeled Reddit posts served as the gold standard corpus for the training and evaluation of various traditional ML and DL algorithms. The best algorithm, one that achieved the highest area under the receiver operating characteristic curve (AUC) [29], was then selected to infer the misinformation label for the remaining unlabeled Reddit posts. Finally, we applied topic modeling to the Reddit posts that were classified as misinformation to explore the major discussion topics and their prevalence.

\section{Data Set Collection and Labeling}

We collected Reddit discussions related to HPV vaccination from 2007 to 2017 ( $\mathrm{N}=28,121)$ using Pushshift [30]. Submissions (topic starters) and comments (responses to the topic) that contained the case-insensitive expressions of cervarix or gardasil or the combination of $h p v$ or papillomavirus with shot or vaccine were collected.
We selected a random sample (simple random sampling) of the total collected Reddit posts $(2200 / 28,121,7.82 \%)$ and labeled the posts as misinformation or nonmisinformation. The purpose of this step was to build a gold standard corpus (ie, Reddit posts with their expert-assigned labels) that was used for the training and evaluation of the automated ML algorithms. The definition of vaccine misinformation was largely informed by the Antivaccination Information class of the Vaccine Misinformation Ontology (VAXMO) [31], a formal ontology to describe vaccine misinformation. Within the VAXMO, the Antivaccination Information class includes several subclasses, such as Vaccine inefficacy, Alternative medicine, Civil liberties, Conspiracy theories, Falsehoods, and Ideological. The random sample of the Reddit posts was used to develop a guideline through discussion among the annotators. A priori consensus was reached among 3 of the study annotators to combine the subclasses Civil Liberties and Ideological and to add two categories: Vaccine recommendations and Other. The resultant decision rules were that if a Reddit post contained one or more types of vaccine misinformation, it was considered an instance of misinformation (Textbox 1).

Textbox 1. Descriptions of types of vaccine misinformation.

- Vaccine inefficacy: vaccine misinformation related to concerns about the lack of effectiveness of vaccines.

- Vaccine safety: vaccine misinformation related to concerns regarding safety issues and supposed harmful ingredients.

- Conspiracy theories: vaccine misinformation related to accusations of a cover-up, where regulatory bodies purportedly have information about vaccines that they are hiding from the public.

- Vaccine recommendations: vaccine misinformation related to vaccine recommendation or schedule.

- Civil liberties and ideologies: the encroachment on personal and parental legal rights or personal principles influencing individual opinions about antivaccine sentiment based on religion, morality, or other ideological reasons.

- Other: other types of vaccine misinformation or a mixed type of misinformation. 
Furthermore, 3 study team members in the fields of biomedical informatics and public health (JD, SP, and HS) were involved in the annotation. The first 100 Reddit posts served as training, with the annotators independently annotating each post and then discussing each post and its annotation as a group. The training ended when the annotators achieved consensus (or took a decision based on a majority vote) on all the posts. After the annotation training, the remaining sampled Reddit posts were split among the 3 annotators for independent labeling. To examine the quality of the annotation, we selected 200 additional posts from the unlabeled Reddit posts, and JD, SP, and HS worked on these posts independently. We calculated the Cohen $\kappa$ among the 3 annotators [32]. The total labeled Reddit samples were used as the basis of a gold standard corpus that was subsequently used for the training and evaluation of the automated ML algorithms.

\section{Misinformation Identification}

\section{Text Classification}

Text classification is a fundamental task of natural language processing (NLP) which aims to classify the textual posts into predefined classes [33]. NLP is a subfield of artificial intelligence that allows computers to process and analyze natural language (ie, free text) data. We framed the identification of misinformation from Reddit posts as a binary text classification task. Each Reddit post was assigned one of two exclusive labels (ie, misinformation or nonmisinformation) within the automated ML-based algorithms (described below).

\section{Algorithms}

We evaluated 5 ML-based algorithms: 3 conventional and 2 DL algorithms. Traditional ML algorithms (ie, nondeep neural network-based algorithms) with feature engineering are widely used for text classification tasks. Altogether, 3 conventional ML algorithms were evaluated in this study: a support vector machine, logistic regression (LR), and extremely randomized trees. Support vector machines have been widely used in text classification tasks [34-36]. LR has achieved favorable performance on many task classification tasks as well but requires substantially less running time [37,38]. Extremely randomized trees is a tree-based ensemble method that has achieved favorable performances in our previous studies on social media text classification tasks [39,40]. Term frequency-inverse document frequency (TF-IDF) was adopted as the feature for these traditional ML algorithms. TF-IDF is a numerical statistic that assesses the relative importance of a word to a document in a corpus [41].

DL is a subset of ML algorithms. We evaluated 2 commonly used DL-based frameworks in this study: convolutional neural network (CNN) [42] and recurrent neural network (RNN) [43]. The effectiveness of traditional ML algorithms depends on task-specific feature engineering [44]. Deep neural networks can take advantage of pretrained word embedding to capture the semantics of words, which saves significant effort in feature engineering by domain experts [45]. DL algorithms have achieved state-of-the-art performance on many text classification tasks [46-49].
As there are frequent occurrences of incorrect spelling in social media posts, both the evaluated DL algorithms contained a character layer and a word-embedding layer to map both in-vocabulary (ie, correctly spelled) and out-of-vocabulary (ie, incorrectly spelled) words to high-dimensional vectors to represent their semantics. GloVe (Global Vectors for Word Representation) embedding (ie, glove.840B.300d) [50] was used to initialize the weights in the word-embedding layer. The CNN model takes word-level embedding as input and feeds it to convolution and max-pooling layers, a fully connected layer and a softmax layer, respectively, for classification [42]. The RNN model follows an architecture that is similar to that of the $\mathrm{CNN}$ model by replacing convolution and max-pooling layers with bidirectional long short-term memory layers and attention layers.

More specifically, for both the CNN and RNN models, the learning rate was set at 0.01 , the batch size was 64 , and the number of epochs was set at 100 . The length of the character embedding was set at 50 for both models. For the CNN model, the filter sizes were 1,2 , and 3 , and the number of filters was 2048; for the RNN model, the hidden dimension of the long short-term memory unit and attention layer was set to 128 . The dropout probability was 0.2 for both models. The model that achieved the best AUC value on the validation set was selected for testing and prediction.

\section{Experiment Settings and Evaluation}

The gold standard posts (ie, Reddit posts with expert-assigned labels) were randomly split into train, validation, and test sets in a ratio of 7:1:2. We adopted spaCy tokenization [51] to split the post text into separate words, remove punctuations, and convert words and letters in uppercase to lowercase. Sequentially, the train set was used to train the algorithms, the validation set was used for hyperparameter selection, and the test set was used to evaluate the performance of the models. To account for imbalance in label distribution, the criterion of reference was the degree of specificity as measured by the optimal AUC. The algorithm with the highest AUC value was selected for the inference of vaccine misinformation from unlabeled Reddit posts.

We further plotted the precision and recall curves of the best-performing model (ie, the CNN model) and selected the optimal cutoff (based on the highest $F_{1}$ score) of the algorithm to identify vaccine misinformation in Reddit posts. Precision was defined as the fraction of misinformation posts identified by the labelers among the fraction of misinformation posts identified by the classifier. Recall was defined as the fraction of misinformation posts identified by the labelers that were retrieved by the classifier. The $F_{1}$ score is a harmonic mean of precision and recall. The cutoff that led to the best $F_{1}$ score for the CNN model was selected. The model was applied to identify vaccine misinformation-related Reddit posts in the remaining unlabeled Reddit corpus.

\section{Misinformation Topic Model}

The ML and DL algorithms described above can potentially be used to automatically identify Reddit posts with misinformation, but they do not categorize the types of misinformation. We 
adopted a topic model algorithm (ie, Biterm Topic model [BTM]) [52], and we implemented the code from a GitHub repository [53]) to identify and visualize major topics from the misinformation in Reddit posts. Topic models are a type of statistical model designed to cluster the abstract topics that occur in a collection of documents. After using the best-performing ML algorithm to identify Reddit posts that contain misinformation, we then applied the BTM to these posts. We performed stemming for each word to remove morphological affixes (eg, dies to die and denied to deni). The number of topics is a hyperparameter for the BTM, which determines the number of topics that will be generated. We evaluated 5, 10, and 20 as the number of topics and selected 10 through a manual review of the topics and associated posts. We then manually reviewed these topics, associated words, and posts to further identify relevant topics associated with vaccine misinformation. The BTM also outputs the prevalence of each identified topic. Word clouds were then adopted to provide a graphic representation of these topics, where the size of each word is proportional to its probability of appearing in posts about that topic [54]. To examine the association of the identified topics, we further performed network analysis among these topics.

\section{Ethics Approval and Consent to Participate}

This study received institutional review board exemption from the committee for the protection of human subjects at the
University of Texas Health Science Center at Houston. The reference number is HSC-SBMI-20-0151.

\section{Results}

\section{Misinformation Annotation}

In total, 28,121 Reddit posts were collected from 2007 to 2017 from more than 16,633 unique users. The statistics of these posts as well as their distributions in subreddits (ie, user-created discussion boards where posts are organized by a subject) are shown in Table 1. There was an increasing trend of HPV vaccine-related discussions (in terms of both the number of posts and number of unique users) during the study period. There were 207,651 upvotes (a user likes the post) and 10,700 downvotes (a user does not like the post) for these posts. Of the 28,121 posts, we manually labeled $2200(7.82 \%)$ randomly selected posts. We measured the annotation agreement by calculating the Cohen $\kappa$ among the 3 annotators: 0.5578 for JD and HS, 0.5216 for JD and SP, and 0.4685 for HS and SP. The agreement scores are considered moderate according to El Eman [32], which indicates a good quality of our gold standard. Among these 2200 posts, 396 (18\%) were annotated as vaccine misinformation, whereas $1804(82 \%)$ were annotated as nonmisinformation. The highly imbalanced label distribution created barriers to achieving high performance for the classification algorithms.

Table 1. The statistics of the human papillomavirus Reddit posts corpus. For statistics regarding Reddit users, we removed the posts if the accounts were unavailable.

\begin{tabular}{|c|c|c|c|c|c|c|c|}
\hline Year & $\begin{array}{l}\text { Total } \\
\text { posts }\end{array}$ & $\begin{array}{l}\text { Total up- } \\
\text { votes }\end{array}$ & $\begin{array}{l}\text { Total down- } \\
\text { votes }\end{array}$ & $\begin{array}{l}\text { Total } \\
\text { unique } \\
\text { users }\end{array}$ & $\begin{array}{l}\text { User posts distribu- } \\
\text { tion, mean (SD) }\end{array}$ & Most frequent subreddits ${ }^{\mathrm{a}}$ (top 3 ) & $\begin{array}{l}\text { Subreddit post distribution, } \\
\text { mean (SD) }\end{array}$ \\
\hline 2007 & 15 & 51 & 1 & 10 & $1.00(0.00)$ & Reddit, 11; politics, 3 ; science, 1 & $5.00(5.29)$ \\
\hline 2008 & 172 & 335 & 35 & 100 & $1.34(1.32)$ & Reddit, 57; science, 54; health, 23 & $11.47(18.88)$ \\
\hline 2009 & 414 & 1563 & 206 & 249 & $1.39(0.93)$ & $\begin{array}{l}\text { Science, } 81 \text {; AskReddit, 51; Reddit, } \\
47\end{array}$ & $14.28(18.96)$ \\
\hline 2010 & 546 & 1655 & 155 & 346 & $1.33(0.79)$ & $\begin{array}{l}\text { AskReddit, 95; sex, 83; TwoXChro- } \\
\text { mosomes, } 72\end{array}$ & $12.13(22.37)$ \\
\hline 2011 & 2156 & 12,711 & 927 & 1382 & $1.37(1.47)$ & $\begin{array}{l}\text { Politics, 298; TwoXChromosomes, } \\
\text { 207; AskReddit, } 203\end{array}$ & $19.42(48.48)$ \\
\hline 2012 & 2457 & 12,812 & 739 & 1641 & $1.32(1.34)$ & $\begin{array}{l}\text { AskReddit, } 457 \text {; TwoXChromosomes, } \\
\text { 308; sex, } 221\end{array}$ & $13.96(48.60)$ \\
\hline 2013 & 3864 & 26,623 & 1416 & 2540 & $1.39(2.44)$ & $\begin{array}{l}\text { Science, 490; AskReddit, 375; sex, } \\
297\end{array}$ & $15.97(51.53)$ \\
\hline 2014 & 3488 & 21,562 & 1581 & 2348 & $1.39(2.62)$ & $\begin{array}{l}\text { Sex, 325; AskReddit, 292; science, } \\
291\end{array}$ & $10.67(34.81)$ \\
\hline 2015 & 4714 & 35,801 & 1761 & 3383 & $1.38(1.61)$ & $\begin{array}{l}\text { News, 378; science, 357; AskReddit, } \\
347\end{array}$ & $11.67(41.28)$ \\
\hline 2016 & 4417 & 38,123 & 1436 & 3137 & $1.39(1.43)$ & $\begin{array}{l}\text { AskReddit, 378; TwoXChromosomes, } \\
\text { 262; sex, } 255\end{array}$ & $10.44(34.25)$ \\
\hline 2017 & 5878 & 56,415 & 2443 & 3752 & $1.56(9.09)$ & AskReddit, 446; sex, 402; news, 387 & $11.28(40.95)$ \\
\hline
\end{tabular}

${ }^{\mathrm{a}}$ Numbers included indicate counts. 


\section{Misinformation Detection and Classification}

The LR algorithm demonstrated the highest AUC value (0.7678) among the 3 traditional ML algorithms used to identify vaccine misinformation in the Reddit posts (Figure 2). Both DL algorithms (CNN and RNN) achieved higher AUC values than the traditional ML algorithms. The CNN model slightly outperformed the RNN model (0.7943 vs 0.7908$)$ in the identification of misinformation. The CNN model with the optimal cutoff was applied to classify the Reddit posts that contained vaccine misinformation. The precision and recall curves of the CNN model are shown in Figure 2. The optimal cutoff led to a precision of 0.4083 , a recall of 0.6202 , and an $F_{1}$ score of 0.4925 . Together with $1.41 \%(396 / 28,121)$ of the Reddit posts that were manually annotated as misinformation, $25.63 \%(7207 / 28,121)$ of the random subset of posts were classified as vaccine misinformation.

Figure 2. The performance of machine learning algorithms on human papillomavirus misinformation identification. (a) Receiver operating characteristic and (b) convolutional neural networks precision-recall curves. AUC: area under the curve; ET: extremely randomized trees; CNN: convolutional neural networks; LR: logistic regression; RNN: recurrent neural network; SVM: support vector machines.

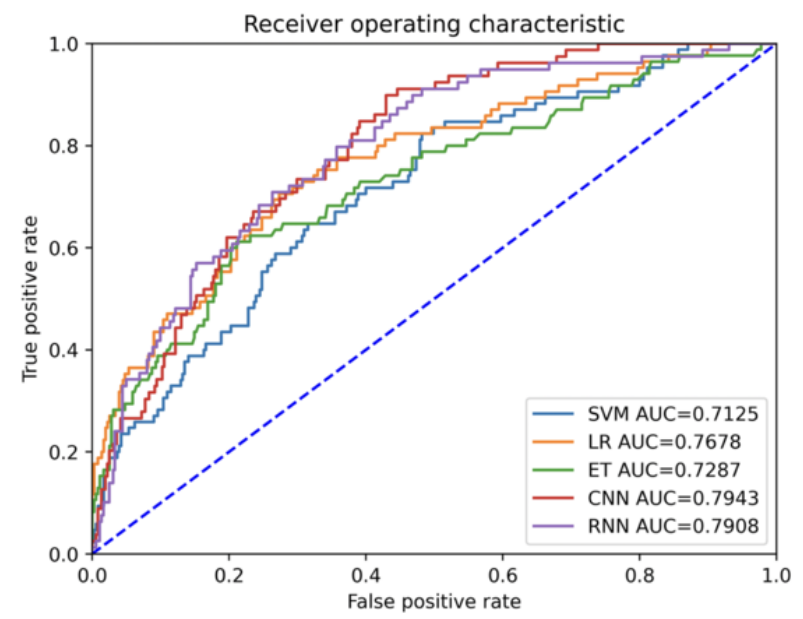

(a)

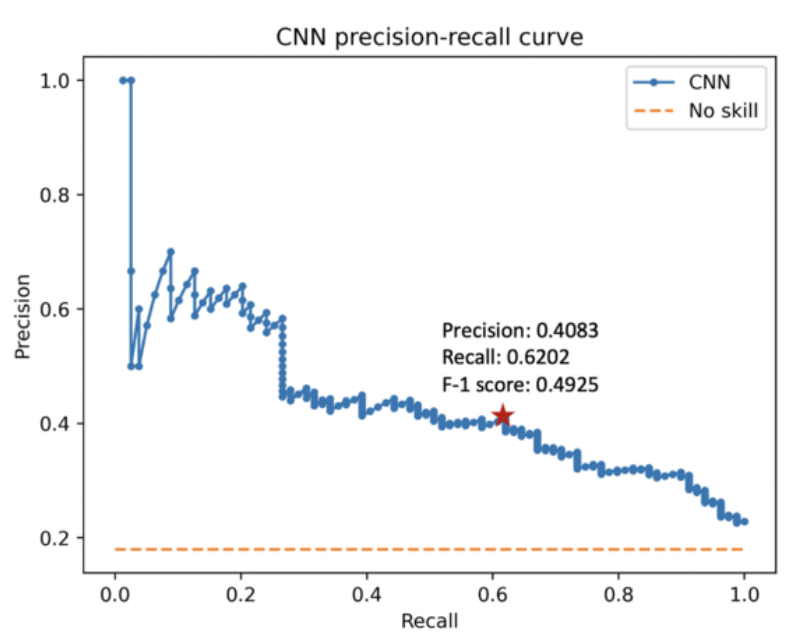

(b)

Topic modeling generated 10 topics from 7207 Reddit posts that were classified as vaccine misinformation. Through qualitative analysis of these 10 algorithm-identified topics and a review of their relevant Reddit posts, we condensed them into 7 (6 major topics + Other) topics. The word clouds for the 6

identified misinformation topics are shown in Figure S1 of Multimedia Appendix 1. The 6 major topics, the percentage of posts assigned to the topic, and post examples are listed in Table 2. 
Table 2. The major topics of misinformation identified by topic modeling $(\mathrm{n}=7207)$.

\begin{tabular}{|c|c|c|c|}
\hline Misinformation topic & Prevalence, n (\%) & Explanation & Examples (excerpts) \\
\hline $\begin{array}{l}\text { General vaccine adverse } \\
\text { events }\end{array}$ & $2672(37.07)$ & $\begin{array}{l}\text { Promotion of general misinforma- } \\
\text { tion regarding the safety of } \mathrm{HPV}^{\mathrm{a}} \\
\text { vaccine }\end{array}$ & $\begin{array}{l}\text { - "The HPV vaccine is unnecessary and unsafe." } \\
\text { - "The HPV vaccination causes retardation." }\end{array}$ \\
\hline Conspiracy theory & $1072(14.87)$ & $\begin{array}{l}\text { Propagation of conspiracy theo- } \\
\text { ries about HPV vaccine and } \\
\text { fraud by the government and } \\
\text { large pharmaceutical companies } \\
\text { (eg, Merck) }\end{array}$ & $\begin{array}{l}\text { - "Rick Perry signed an executive order trying to mandate } \\
\text { Gardasil vaccines for young girls. He did this to make } \\
\text { money for his buddies at Merck." } \\
\text { "The HPV vaccine is a joke pressed on schools and admin- } \\
\text { istrations by greedy pharmaceutical corporations." }\end{array}$ \\
\hline Citing unfounded studies & $989(13.72)$ & $\begin{array}{l}\text { This type of misinformation can } \\
\text { be very misleading because it } \\
\text { tends to cite and interpret scien- } \\
\text { tific studies from sources that are } \\
\text { not scientifically peer reviewed }\end{array}$ & $\begin{array}{l}\text { - "Flawed safety study on HPV vaccine triggers butterfly ef- } \\
\text { fect - leaked e-mails reveal who suppressed info on danger- } \\
\text { ous particles in vaccine" } \\
\text { "We find that HPV vaccine clinical trials design, and data } \\
\text { interpretation of both efficacy and safety outcomes, were } \\
\text { largely inadequate" }\end{array}$ \\
\hline $\begin{array}{l}\text { Vaccine deaths and serious } \\
\text { reactions }\end{array}$ & $520(7.21)$ & $\begin{array}{l}\text { Propagation of HPV vaccine-in- } \\
\text { duced death and serious adverse } \\
\text { reactions }\end{array}$ & $\begin{array}{l}\text { - "For instance, the HPV vaccine has caused children to die." } \\
\text { "The shots have killed women. The adverse event reports } \\
\text { from the FDA }{ }^{\text {b }} \text { about HPV vaccination read like a litany of } \\
\text { horrors." }\end{array}$ \\
\hline $\begin{array}{l}\text { Aluminum-containing ad- } \\
\text { juvants }\end{array}$ & $456(6.33)$ & $\begin{array}{l}\text { Promoting misinformation on } \\
\text { safety issues of aluminum-con- } \\
\text { taining adjuvants in vaccines }\end{array}$ & $\begin{array}{l}\text { - } \quad \text { Another good keyword is for Gardasil adverse drug reac- } \\
\text { tions. That's how I found this: 'each } 0.5 \text {-ml dose of the } \\
\text { vaccine contains approximately } 225 \mathrm{mcg} \text { of aluminum (as } \\
\text { amorphous aluminum hydroxyphosphate sulfate adjuvant)' } \\
\text { This study clearly shows that aluminum found in vaccines } \\
\text { can cause neurologic damage." } \\
\text { "Brain damage and autoimmune diseases can be caused by } \\
\text { aluminum adjuvants. Aluminum adjuvant is in the HPV } \\
\text { vaccine." }\end{array}$ \\
\hline Vaccine and autism & $198(2.75)$ & $\begin{array}{l}\text { Promoting misinformation on the } \\
\text { discredited links between vaccine } \\
\text { and autism }\end{array}$ & $\begin{array}{l}\text { - "The HPV vaccine causes autism" } \\
\text { "Vaccine court awarded millions to two children with } \\
\text { autism. CDC }{ }^{\mathrm{c}} \text { report creates controversy for Merck's Gar- } \\
\text { dasil vaccine" }\end{array}$ \\
\hline
\end{tabular}

${ }^{\mathrm{a}} \mathrm{HPV}$ : human papillomavirus.

${ }^{b}$ FDA: Food and Drug Administration.

${ }^{\mathrm{c}} \mathrm{CDC}$ : US Centers for Disease Control and Prevention.

\section{Misinformation Network Analysis}

We further analyzed the network among the identified topics. For each Reddit post, we identified the 2 most associated topics (ranked by probability generated by the BTM). We assume that these top 2 topics were linked for that post, which was considered an undirected edge in the network. Figure S2 in Multimedia Appendix 1 shows the misinformation topic network among these 7 topics. The size of the circle for each topic is proportional to the degree of associations of the topic (ie, the number of connections with other topics). The width of the edge is proportional to the number of connections between the 2 topics.

\section{Discussion}

\section{Principal Findings}

In this study, we evaluated the use of different ML-based approaches to analyze Reddit discussions related to HPV vaccine misinformation. The CNN and RNN algorithms improved the
AUC value compared with the traditional ML algorithms. A BTM was adopted to further explore the major topics related to vaccine misinformation discussions. Overall, 6 major topics related to HPV vaccine misinformation, including Vaccine death and serious reactions and Aluminum-containing adjuvants were identified. The Vaccine adverse effect, which refers to general misinformation regarding safety issues, is the most prevalent topic within HPV vaccine misinformation.

The highest proportion of vaccine misinformation content on Reddit identified with our approach concerned general vaccine adverse effects $(2672 / 7207,37.07 \%)$, followed by content about vaccine conspiracy theories $(1072 / 7207,14.87 \%)$. These results are consistent with previous analyses of social media-based vaccine misinformation, which found that inaccuracies about vaccine knowledge and risk (37.9\%) made up most of the social media posts with negative vaccine sentiments [55]. The same small study also found that $13.8 \%$ of the negative posts about vaccines included distrust of government and pharmaceutical 
companies, which closely mirrors our findings in a larger sample from Reddit.

We further analyzed the top subreddits among the posts that ML inferred as containing misinformation and nonmisinformation. The subreddits that contain the most misinformation-related posts include science $(\mathrm{n}=653)$, AskReddit $(\mathrm{n}=604)$, conspiracy $(\mathrm{n}=593)$, and politics $(\mathrm{n}=397)$. On the contrary, the subreddits that contain the most nonmisinformation-related posts include AskReddit $(\mathrm{n}=2040)$, sex $(\mathrm{n}=1994)$, TwoXChromosomes $(\mathrm{n}=1652)$, and science $(\mathrm{n}=1385)$. Besides general popular subreddits such as science and AskReddit, misinformation tends to cluster in subreddits such as conspiracy and politics. There is an increasing trend of discussing HPV vaccine-related topics on Reddit from 15 posts in 2007 to 5878 posts in 2017. There is a decreasing trend in the proportion of misinformation over time on Reddit. The proportion of misinformation ranged from $41.8 \%$ (72/172; in year 2008) to $53 \%$ (8/15; in year 2007) during the period 2007 to 2009 , whereas it ranged from $22.84 \%$ (1009/4417; in year $2016)$ to $33.85 \%(730 / 2156$; in year 2011$)$ during the period 2010 to 2017 . The decrease could be a result of the continuous promotion efforts made by public health professionals, as well as an increase in internet verification skills among users.

The results of our network analysis of the 6 identified vaccine misinformation topics (in addition to Other) further reinforce our findings and demonstrate the strength of the connectedness of each topic. Although general concerns about the safety of the vaccine emerged as the main source of hesitancy regarding HPV vaccination, the network analysis indicates that the other prominent topics identified, such as the presence of conspiracy theories, may also be rooted in fears about the side effects of the vaccine. Mere exposure to beliefs that the government and pharmaceutical companies gain or profit from mass vaccination through deception or at the consumers' expense, has strong negative effects on attitudes about the safety and effectiveness of vaccines, consequently affecting choices about whether to vaccinate [56].

Of note, the annotators anecdotally observed that the Reddit posts identified in this study did not seem to be connected to any organized movements; rather, they were by single users advocating their personal views. A potential method to combat these misinformed messages once identified is to counter them with an organized campaign, composed of factual, evidence-based messages, that does not acknowledge disinformation. As other studies have noted, acknowledging and deferring to web-based disputes related to vaccines may cause health information seekers to doubt established evidence regarding vaccine efficacy and safety [57]. In addition, it has been found that attempting to correct misinformation directly often reinforces the sentiments of those holding strong antivaccine views [58].

To the best of our knowledge, this is one of the early efforts to explore the use of automated ML algorithms (eg, ML and NLP) to identify and classify HPV vaccine misinformation in social media discussions. We chose the HPV vaccine as the use case for our analysis, but the proposed methodology framework can also be applied to other types of vaccines or other pertinent health-related topics. The ML-based framework is also scalable to big social media data. Our work could assist policy makers and the industry to accurately understand and address the spread of health misinformation on social media. The methodology framework developed in this study is generalizable to other social media platforms such as Twitter and can be used to identify misinformation in both retrospective and real-time social media feeds. The use of this methodology could be incorporated into social media platforms dedicated to curbing the spread of health-related misinformation on these sites, although the ethical ramifications of such restrictions should be taken into consideration.

\section{Limitations and Future Work}

This study should be interpreted in light of limitations and future research needs. Given the unique features of social media language, the accurate identification of misinformation is a very challenging task. The best algorithm achieved an AUC value of 0.7943 , and there is some room for improving this performance. Our current ML classifier has a higher recall than precision (0.6202 vs 0.4083$)$. This means that the classifier tends to label both misinformation and nonmisinformation as misinformation. In a real-world scenario, the classifier may serve as a tool to prescreen misinformation, and more rigorous fact-checking methods (eg, human checking) would be needed to label true misinformation posts. The high imbalanced label distribution (ie, only 396/2200, 18\%) of the posts were labeled as misinformation in the gold standard corpus) hurt the ML algorithm because most of the ML algorithms used for classification were designed based on the assumption of an equal number of examples for each class. Imbalanced label distribution results in models that have poor predictive performance, specifically for the minority class (eg, misinformation in our case) [59]. As we further refine and expand the gold standard corpus, which is critical for the evaluation and training of ML algorithms, we expect the performance to improve. In addition, we will explore the use of data augmentation techniques [60] and random oversampling methods $[61,62]$ to alleviate the issues caused by imbalanced label distribution. Other emerging advanced DL algorithms such as Bidirectional Encoder Representations from Transformers (BERT) [63] hold promise for improved performance. In addition, we performed annotation at the level of Reddit posts, which may have sacrificed precision. A single Reddit post often contains multiple sentences, allowing a mix of misinformation and nonmisinformation to exist in a single post. Therefore, a Reddit post annotated as misinformation could also contain evidence-based facts. Future research can establish the effect of annotation and classification at the sentence level to improve the precision of misinformation identification. In addition, the identification of abstract topics from topic modeling is a semiautomated process combined with expert review. However, topic assignment and summarization may be subjective and suffer from biases as well. In future, we can explore the use of supervised algorithms for more precise topic discovery. 


\section{Conclusions}

Our ML-based approaches demonstrated efficacy in the automated identification and classification of HPV vaccine misinformation in discussions on the social media platform Reddit. The large quantity of web- and social media-based medical and public health information available may make it difficult for those with low health and web literacy to navigate and find authentic and evidence-based information. Although our ML algorithm does not solve the problem of health and vaccine misinformation single-handedly, we provide an innovative stepping stone that may bridge multiple approaches for combating this invasive and growing public health concern. The accurate and timely understanding of vaccine misinformation on social media can assist vaccine promotion campaigns to prevent such information from misleading the vulnerable public. Our methodology could also be applied to other social media platforms such as Twitter, although new labeled data would be necessary.

\section{Acknowledgments}

This research was supported by the National Institutes of Health under award numbers 2R01LM010681-05, R01 LM011829, and NCI T32/CA057712 (SP); and the Cancer Prevention and Research Institute of Texas training grant \#RP160015. The content is solely the responsibility of the authors and does not necessarily represent the official views of the National Library of Medicine and the Cancer Prevention and Research Institute of Texas.

\section{Authors' Contributions}

JD and CT have full access to all data in the study and take responsibility for the integrity of the data and the accuracy of the data analysis. Study concept and design was conducted by JD, SP, MA, and CT. Data annotation was carried out by JD, SP, and HS. JD, SP, RS, MA, and CT were involved in drafting of the manuscript. Acquisition, analysis, or interpretation of data was conducted by JD, SP, MA, and CT. Critical revision of the manuscript for important intellectual content was carried out by all authors. Study supervision was conducted by CT.

\section{Conflicts of Interest}

None declared.

\section{Multimedia Appendix 1}

Supplemental tables and figures.

[DOCX File, 1055 KB-Multimedia Appendix 1]

\section{References}

1. Human Papillomavirus (HPV) - reasons to get vaccinated. Centers for Disease Control and Prevention. 2019. URL: https:/ /www.cdc.gov/hpv/parents/vaccine/six-reasons.html [accessed 2021-07-02]

2. Saraiya M, Unger E, Thompson T, Lynch CF, Hernandez BY, Lyu CW, HPV Typing of Cancers Workgroup. US assessment of HPV types in cancers: implications for current and 9-valent HPV vaccines. J Natl Cancer Inst 2015 Jun;107(6):djv086 [FREE Full text] [doi: 10.1093/jnci/djv086] [Medline: 25925419]

3. HPV vaccine: who needs it, how it works. Mayo Clinic. 2019. URL: https://www.mayoclinic.org/diseases-conditions/ hpv-infection/in-depth/hpv-vaccine/art-20047292 [accessed 2020-02-09]

4. Zimet GD, Rosberger Z, Fisher WA, Perez S, Stupiansky NW. Beliefs, behaviors and HPV vaccine: correcting the myths and the misinformation. Prev Med 2013 Nov;57(5):414-418 [FREE Full text] [doi: 10.1016/j.ypmed.2013.05.013] [Medline: 23732252]

5. Etter DJ, Zimet GD, Rickert VI. Human papillomavirus vaccine in adolescent women: a 2012 update. Curr Opin Obstet Gynecol 2012 Oct;24(5):305-310. [doi: 10.1097/GCO.0b013e3283567005] [Medline: 22781077]

6. Holman DM, Benard V, Roland KB, Watson M, Liddon N, Stokley S. Barriers to human papillomavirus vaccination among US adolescents: a systematic review of the literature. JAMA Pediatr 2014 Jan 01;168(1):76-82 [FREE Full text] [doi: 10.1001/jamapediatrics.2013.2752] [Medline: 24276343]

7. Poland G, Jacobson R. Understanding those who do not understand: a brief review of the anti-vaccine movement. Vaccine 2001 Mar;19(17-19):2440-2445. [doi: 10.1016/s0264-410x(00)00469-2]

8. Geoghegan S, O'Callaghan KP, Offit PA. Vaccine safety: myths and misinformation. Front Microbiol 2020 Mar 17;11:372 [FREE Full text] [doi: 10.3389/fmicb.2020.00372] [Medline: $\underline{32256465]}$

9. Kata A. Anti-vaccine activists, web 2.0, and the postmodern paradigm--an overview of tactics and tropes used online by the anti-vaccination movement. Vaccine 2012 May 28;30(25):3778-3789. [doi: 10.1016/j.vaccine.2011.11.112] [Medline: 22172504]

10. Kata A. A postmodern Pandora's box: anti-vaccination misinformation on the internet. Vaccine 2010 Feb 17;28(7):1709-1716. [doi: 10.1016/j.vaccine.2009.12.022] [Medline: 20045099]

11. Anderson J, Rainie L. Experts optimistic about the next 50 years of digital life. Pew Research Center. 2019. URL: https:/ /www.pewresearch.org/internet/2019/10/28/experts-optimistic-about-the-next-50-years-of-digital-life/ [accessed 2021-07-16] 
12. Levin S. Facebook promised to tackle fake news. But the evidence shows it's not working. The Guardian. 2017. URL: https://www.theguardian.com/technology/2017/may/16/facebook-fake-news-tools-not-working [accessed 2020-02-10]

13. Ghosh D, Scott B. Disinformation is becoming unstoppable. Time. 2018. URL: https://time.com/5112847/ facebook-fake-news-unstoppable/ [accessed 2020-02-10]

14. Moorhead SA, Hazlett DE, Harrison L, Carroll JK, Irwin A, Hoving C. A new dimension of health care: systematic review of the uses, benefits, and limitations of social media for health communication. J Med Internet Res 2013 Apr;15(4):e85 [FREE Full text] [doi: 10.2196/jmir.1933] [Medline: 23615206]

15. Chou WS, Oh A, Klein WM. Addressing health-related misinformation on social media. J Am Med Assoc 2018 Dec 18;320(23):2417-2418. [doi: 10.1001/jama.2018.16865] [Medline: $\underline{30428002}$ ]

16. Qazvinian V, Rosengren E, Radev D, Mei Q. Rumor has it: identifying misinformation in microblogs. In: Proceedings of the 2011 Conference on Empirical Methods in Natural Language Processing. 2011 Presented at: Conference on Empirical Methods in Natural Language; July, 2011; Edinburgh, Scotland, UK p. 1589-1599 URL: https://aclanthology.org/D11-1147

17. Hamidian S, Diab M. Rumor detection and classification for Twitter data. arXiv. URL: http://arxiv.org/abs/1912.08926 [accessed 2021-07-02]

18. Machine learning. Wikipedia. URL: https://en.wikipedia.org/wiki/Machine learning [accessed 2020-02-09]

19. Ghenai A, Mejova Y. Catching Zika fever: application of crowdsourcing and machine learning for tracking health misinformation on Twitter. In: Proceedings of the IEEE International Conference on Healthcare Informatics (ICHI). 2017 Presented at: IEEE International Conference on Healthcare Informatics (ICHI); Aug. 23-26, 2017; Park City, UT, USA. [doi: 10.1109/ichi.2017.58]

20. Kinsora A, Barron K, Mei Q, Vydiswaran V. Creating a labeled dataset for medical misinformation in health forums. In: Proeedings of the IEEE International Conference on Healthcare Informatics (ICHI). 2017 Presented at: IEEE International Conference on Healthcare Informatics (ICHI); Aug. 23-26, 2017; Park City, UT, USA p. 456-461. [doi: 10.1109/ichi.2017.93]

21. Du J, Xu J, Song H, Liu X, Tao C. Optimization on machine learning based approaches for sentiment analysis on HPV vaccines related tweets. J Biomed Semantics 2017 Mar 03;8(1):9 [FREE Full text] [doi: 10.1186/s13326-017-0120-6] [Medline: 28253919]

22. Zhou X, Coiera E, Tsafnat G, Arachi D, Ong M, Dunn A. Using social connection information to improve opinion mining: identifying negative sentiment about HPV vaccines on Twitter. Stud Health Technol Inform 2015;216:761-765. [doi: 10.3233/978-1-61499-564-7-761]

23. LeCun Y, Bengio Y, Hinton G. Deep learning. Nature 2015 May 28;521(7553):436-444. [doi: 10.1038/nature14539] [Medline: 26017442]

24. Mediakix. 2018. URL: https://mediakix.com/blog/reddit-statistics-users-demographics/ [accessed 2020-02-05]

25. Frequently asked questions. National Institute for Communicable Diseases. URL: https://www.nicd.ac.za/diseases-a-z-index/ covid-19/frequently-asked-questions/ [accessed 2018-12-04]

26. Barnard M, George P, Perryman ML, Wolff LA. Human papillomavirus (HPV) vaccine knowledge, attitudes, and uptake in college students: implications from the Precaution Adoption Process Model. PLoS One 2017 Aug 7;12(8):e0182266 [FREE Full text] [doi: 10.1371/journal.pone.0182266] [Medline: 28786994]

27. Thompson EL, Vamos CA, Vázquez-Otero C, Logan R, Griner S, Daley EM. Trends and predictors of HPV vaccination among U.S. college women and men. Prev Med 2016 May;86:92-98. [doi: 10.1016/j.ypmed.2016.02.003] [Medline: 26868093]

28. Abrol E, Groszmann M, Pitman A, Hough R, Taylor RM, Aref-Adib G. Exploring the digital technology preferences of teenagers and young adults (TYA) with cancer and survivors: a cross-sectional service evaluation questionnaire. J Cancer Surviv 2017 Dec;11(6):670-682 [FREE Full text] [doi: 10.1007/s11764-017-0618-z] [Medline: 28634734]

29. Sedgwick P. Receiver operating characteristic curves. Br Med J 2011 Jul 13;343(jul13 2):d4302. [doi: 10.1136/bmj.d4302]

30. Pushshift.io. URL: https://pushshift.io/ [accessed 2021-07-16]

31. Amith M, Tao C. Representing vaccine misinformation using ontologies. J Biomed Semantics 2018 Aug 31;9(1):22 [FREE Full text] [doi: 10.1186/s13326-018-0190-0] [Medline: 30170633]

32. Emam EK. Benchmarking Kappa: interrater agreement in software process assessments. Empir Softw Eng 1999;4(2):113-133. [doi: 10.1023/A:1009820201126]

33. Jurafsky D, Martin JH. Speech and language processing 2nd Edition. Upper Saddle River, New Jersey: Prentice-Hall, Inc; 2009.

34. Naz S, Sharan A, Malik N. Sentiment classification on Twitter data using support vector machine. In: Proceedings of the IEEE/WIC/ACM International Conference on Web Intelligence (WI). 2018 Presented at: IEEE/WIC/ACM International Conference on Web Intelligence (WI); Dec. 3-6, 2018; Santiago, Chile. [doi: 10.1109/wi.2018.00-13]

35. Zhang W, Yoshida T, Tang X. Text classification based on multi-word with support vector machine. Knowledge-Based Systems 2008 Dec;21(8):879-886. [doi: 10.1016/j.knosys.2008.03.044]

36. Tong S, Koller D. Support vector machine active learning with applications to text classification. J Mach Learn Res. (Nov) 2001;2:45-66 [FREE Full text] 
37. Pranckevičius T, Marcinkevičius V. Comparison of naive bayes, random forest, decision tree, support vector machines, and logistic regression classifiers for text reviews classification. Balt J Mod Comput 2017;5(2):221. [doi: 10.22364/bjmc.2017.5.2.05]

38. Aborisade O, Anwar M. Classification for authorship of tweets by comparing logistic regression and naive bayes classifiers. In: Proceedings of the IEEE International Conference on Information Reuse and Integration (IRI). 2018 Presented at: IEEE International Conference on Information Reuse and Integration (IRI); July 6-9, 2018; Salt Lake City, UT, USA. [doi: 10.1109/iri.2018.00049]

39. Du J, Luo C, Shegog R, Bian J, Cunningham RM, Boom JA, et al. Use of deep learning to analyze social media discussions about the human papillomavirus vaccine. JAMA Netw Open 2020 Nov 02;3(11):e2022025 [FREE Full text] [doi: 10.1001/jamanetworkopen.2020.22025] [Medline: 33185676]

40. Du J, Cunningham RM, Xiang Y, Li F, Jia Y, Boom JA, et al. Leveraging deep learning to understand health beliefs about the Human Papillomavirus Vaccine from social media. NPJ Digit Med 2019 Apr 15;2(1):27. [doi: 10.1038/s41746-019-0102-4]

41. Ramos J. Using TF-IDF to determine word relevance in document queries. In: Proceedings of the First Instructional Conference on Machine Learning. 2003 Presented at: First Instructional Conference on Machine Learning; June 23-24, 2003; Los Angeles, California p. 29-48 URL: http://citeseerx.ist.psu.edu/viewdoc/download?doi=10.1.1.121. $1424 \&$ rep=rep1\&type=pdf

42. Du J, Luo C, Wei Q, Chen Y, Tao C. Exploring difference in public perceptions on HPV vaccine between gender groups from Twitter using deep learning. arXiv. 2019. URL: http://arxiv.org/abs/1907.03167 [accessed 2021-07-02]

43. Zhou P, Shi W, Tian J, Qi Z, Li B, Hao H, et al. Attention-based bidirectional long short-term memory networks for relation classification. In: Proceedings of the 54th Annual Meeting of the Association for Computational Linguistics. 2016 Presented at: 54th Annual Meeting of the Association for Computational Linguistics; Augut, 2016; Berlin, Germany p. 207-212. [doi: $10.18653 / \mathrm{v} 1 / \mathrm{p} 16-2034]$

44. Kharde VA, Sonawane SS. Sentiment Analysis of Twitter Data: A Survey of Techniques. IJCA 2016 Apr 15;139(11):5-15. [doi: 10.5120/ijca2016908625]

45. Young T, Hazarika D, Poria S, Cambria E. Recent trends in deep learning based natural language processing [Review article]. IEEE Comput Intell Mag 2018 Aug;13(3):55-75. [doi: 10.1109/MCI.2018.2840738]

46. Zhang L, Wang S, Liu B. Deep learning for sentiment analysis: a survey. WIREs Data Mining Knowl Discov 2018 Mar 30;8(4):e1253. [doi: 10.1002/widm.1253]

47. Zhang X, Zhao J, Lecun Y. Character-level convolutional networks for text classification. In: Advances in Neural Information Processing Systems. Cambridge, MA: MIT Press; 2015:649-657 URL: https://proceedings.neurips.cc/paper/2015/file/ 250cf8b51c773f3f8dc8b4be867a9a02-Paper.pdf

48. Liu G, Guo J. Bidirectional LSTM with attention mechanism and convolutional layer for text classification. Neurocomputing 2019 Apr;337:325-338. [doi: 10.1016/j.neucom.2019.01.078]

49. Liu X, You X, Zhang X, Wu J, Lv P. Tensor graph convolutional networks for text classification. arXiv. 2020. URL: https:/ /arxiv.org/abs/2001.05313 [accessed 2021-07-02]

50. Pennington J, Socher R, Manning C. GloVe: Global vectors for word representation. In: Proceedings of the 2014 Conference on Empirical Methods in Natural Language Processing (EMNLP). 2014 Presented at: EMNLP Conference on Empirical Methods in Natural Language Processing; October, 2014; Doha, Qatar p. 1532-1543. [doi: 10.3115/v1/d14-1162]

51. Tokenizer. spaCy API Documentation. URL: https://spacy.io/api/tokenizer [accessed 2020-05-29]

52. Yan X, Guo J, Lan Y, Cheng X. A biterm topic model for short texts. In: Proceedings of the 22nd International Conference on World Wide Web. 2013 Presented at: WWW '13: 22nd International World Wide Web Conference; May 13-17, 2013; Rio de Janeiro, Brazil p. 1445-1456. [doi: 10.1145/2488388.2488514]

53. xiaohuiyan/BTM: Code for Biterm Topic Model. Github. URL: https://github.com/xiaohuiyan/BTM [accessed 2020-03-05]

54. Generating word cloud in Python. GeeksforGeeks. URL: https://www.geeksforgeeks.org/generating-word-cloud-python/ [accessed 2020-06-08]

55. Tustin JL, Crowcroft NS, Gesink D, Johnson I, Keelan J, Lachapelle B. User-driven comments on a Facebook advertisement recruiting Canadian parents in a study on immunization: content analysis. JMIR Public Health Surveill 2018 Sep 20;4(3):e10090 [FREE Full text] [doi: 10.2196/10090] [Medline: 30249585]

56. Jolley D, Douglas KM. The effects of anti-vaccine conspiracy theories on vaccination intentions. PLoS One 2014 Feb 20;9(2):e89177 [FREE Full text] [doi: 10.1371/journal.pone.0089177] [Medline: 24586574]

57. Dixon $\mathrm{G}$, Clarke $\mathrm{C}$. The effect of falsely balanced reporting of the autism-vaccine controversy on vaccine safety perceptions and behavioral intentions. Health Educ Res 2013 Apr 27;28(2):352-359. [doi: 10.1093/her/cys 110] [Medline: 23193194]

58. Nyhan B, Reifler J. When corrections fail: the persistence of political misperceptions. Polit Behav 2010 Mar 30;32(2):303-330. [doi: 10.1007/s11109-010-9112-2]

59. Krawczyk B. Learning from imbalanced data: open challenges and future directions. Prog Artif Intell 2016 Apr 22;5(4):221-232. [doi: 10.1007/s13748-016-0094-0]

60. Wei J, Zou K. EDAasy data augmentation techniques for boosting performance on text classification tasks. In: Proceedings of the 2019 Conference on Empirical Methods in Natural Language Processing and the 9th International Joint Conference 
on Natural Language Processing (EMNLP-IJCNLP). 2019 Presented at: Conference on Empirical Methods in Natural Language Processing and the 9th International Joint Conference on Natural Language Processing (EMNLP-IJCNLP); November, 2019; Hong Kong, China p. 6382-6388. [doi: 10.18653/v1/d19-1670]

61. Moreo A, Esuli A, Sebastiani F. Distributional random oversampling for imbalanced text classification. In: Proceedings of the 39th International ACM SIGIR Conference on Research and Development in Information Retrieval. 2016 Presented at: SIGIR '16: The 39th International ACM SIGIR Conference on Research and Development in Information Retrieval; July 17-21, 2016; Pisa Italy p. 805-808. [doi: 10.1145/2911451.2914722]

62. Charte F, Rivera AJ, del Jesus MJ, Herrera F. Addressing imbalance in multilabel classification: measures and random resampling algorithms. Neurocomputing 2015 Sep;163:3-16. [doi: 10.1016/j.neucom.2014.08.091]

63. Devlin J, Chang M, Lee K, Toutanova K. BERT: Pre-training of Deep Bidirectional Transformers for Language Understanding. arXiv preprint arXiv:1810.04805. 2018 Oct 11. URL: $\underline{\text { https://arxiv.org/abs/1810.04805 }}$

\author{
Abbreviations \\ AUC: area under the receiver operating characteristic curve \\ BERT: Bidirectional Encoder Representations from Transformers \\ BTM: Biterm Topic model \\ CNN: convolutional neural network \\ DL: deep learning \\ GloVe: Global Vectors for Word Representation \\ HPV: human papillomavirus \\ LR: logistic regression \\ ML: machine learning \\ NLP: natural language processing \\ RNN: recurrent neural network \\ TF-IDF: term frequency-inverse document frequency \\ VAXMO: Vaccine Misinformation Ontology
}

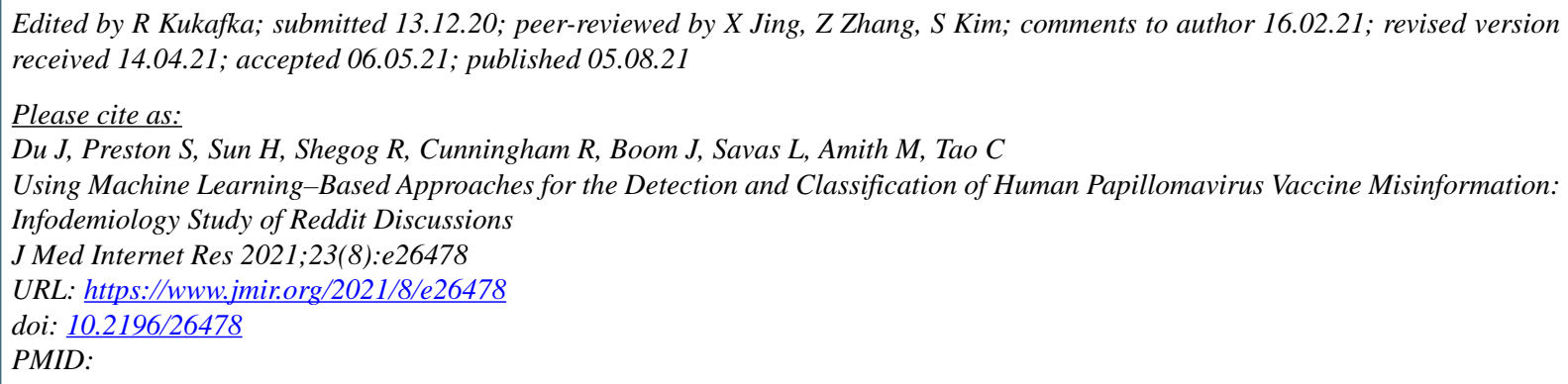

CJingcheng Du, Sharice Preston, Hanxiao Sun, Ross Shegog, Rachel Cunningham, Julie Boom, Lara Savas, Muhammad Amith, Cui Tao. Originally published in the Journal of Medical Internet Research (https://www.jmir.org), 05.08.2021. This is an open-access article distributed under the terms of the Creative Commons Attribution License (https://creativecommons.org/licenses/by/4.0/), which permits unrestricted use, distribution, and reproduction in any medium, provided the original work, first published in the Journal of Medical Internet Research, is properly cited. The complete bibliographic information, a link to the original publication on https://www.jmir.org/, as well as this copyright and license information must be included. 